\title{
SELF SHADOWS AND CAST SHADOWS IN ESTIMATING ILLUMINATION DISTRIBUTION
}

\author{
Takeshi Takai, Atsuto Maki, Takashi Matsuyama \\ Graduate School of Informatics, Kyoto University, Kyoto, Japan. \\ \{takesi-t, maki, tm\}@ vision.kuee.kyoto-u.ac.jp
}

Keywords: Illumination estimation, Near lighting, Dynamic lighting, Self shadows, Skeleton cube

\begin{abstract}
This paper compares two methods for estimating illumination distributions from shadows: methods using self shadows and cast shadows. The cast shadow method provides an effective framework for recovering illuminations in a real scene utilizing variations of image brightnesses inside shadows cast by an occluding object of known shape. The shadow surface is typically the same plane on which the object is placed. A new self shadow method is proposed in this paper in which the shadow surface is on the occluding object itself. We show that it works even for lighting environments where the cast shadow method fails while clarifying the stability issues related to sampling resolution, and the selection of lighting model. We also present an automatic framework for estimation of near lighting by a depth identification, as well as the directional analysis of the illumination distribution, which was not addressed in the cast shadow method. We prove the effectiveness of our new method with experiments using real scenes.
\end{abstract}

\section{Introduction}

Lighting environment estimation is one of the most important research topics in computer vision. If it is possible to derive the lighting information from an input image of a real scene, it enables us to superimpose virtual objects into the scene with natural shading and shadows by controlling the lighting effects in the generated or captured images accordingly (Figure 1). The applications of lighting environment estimation are widespread, such as object tracking, face recognition, VR and graphics.

For lighting environment estimation various methods, either direct or indirect, have been proposed. Pioneering work includes those of estimating illuminant directions in the framework of shape from shading [7, 18]. In direct sensing such as in $[1,11]$, images of the illuminant distribution are directly captured by a camera, and the lighting environment is estimated by analyzing the pixel values of the images. Among indirect sensing approaches some exploit the occluding boundary that puts strong constraints on the light source directions using an object that has a (locally) Lambertian surface $[6,16]$. Some other indirect approaches [5, 15, 17, 19],

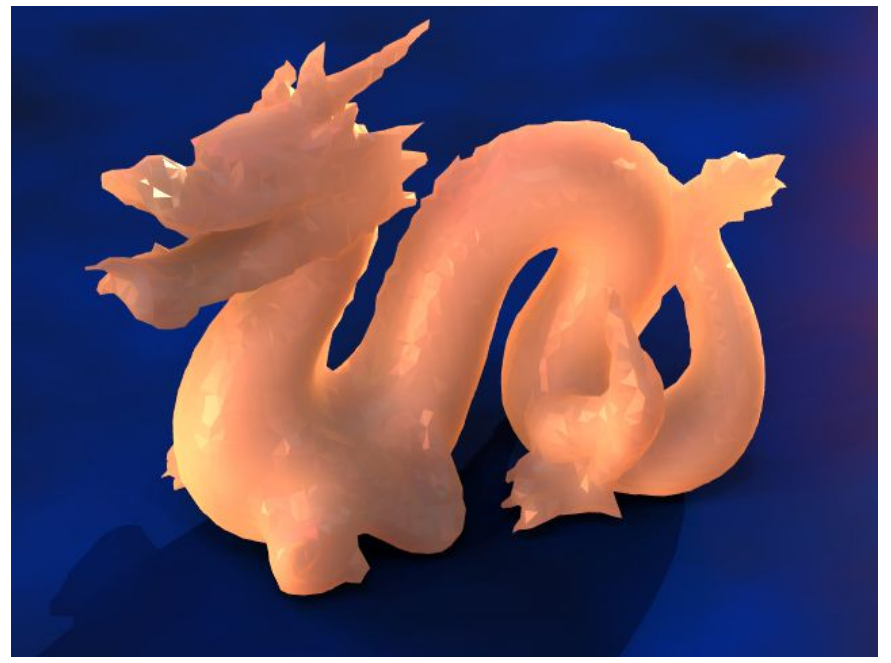

Figure 1: CG image rendered under estimated illumination by the proposed method. The illumination is represented by a distribution of near light sources.

so-called inverse lighting, employ images containing a reference object with known shape and reflectance properties, and estimate the lighting environment by analyzing its shading or highlights.

Recently, Sato et al. have introduced a method for inverse lighting [12], in which the lighting environment is characterized by a distributed set of directional light sources; their radiant intensities can be efficiently estimated by analyzing pixel values of shadows on a plane cast by an object of known shape. We refer to the method as the cast shadow method in this paper. As pointed out in [10], however, a natural question to the method is whether a given input image always provides sufficient information to robustly estimate the illumination distribution. Namely, it is discussed that possible instability of estimation is due to factors such as what size portions of shadows cast by an object can be observed in the input image, and what size portions of the view of the camera are occluded by the object.

To enlarge the variety of images to which the method can be applied, some efficient strategies including the techniques of changing the sampling density of illumination distribution or optimal sampling of image pixels have also been introduced [10]. Despite such advances, the above mentioned factors still remain open issues. This is the case especially when the 
proximity of light source is to be considered, i.e., the closer a light source is to the occluding object, the larger portion of shadow is likely to be cast by the object, which causes such instability as previously discussed. Although a few recent advances $[3,9,13,2]$ allow estimation of near light source, it is still a challenging problem to solve for parameters of a complex lighting environment.

In this paper, based on the promising framework of the cast shadow method, we propose the self shadow method in which the shadow surface is on the occluding object itself. As a possible occluding object, we utilize a hollow cube with specific shape, the Skeleton Cube (see Figure 2) which allows self shadows to occur in a consistent way. We then analyze the pixel values of shading and self shadows on its inside surfaces for estimating radiant intensities of distributed light sources.

Comparing the cast shadow method and the self shadow method, we clarify the limitation of the former, and show why the latter works even for lighting environments that the former fails to estimate. Since self shadows are observed on the inside surface of the skeleton cube under light sources at almost any positions, in practice, it is convenient that we have only to examine its inside surfaces without worrying about the observable size portions of shadows in the input image. Finally, we also present an automatic framework for estimating near lighting by a depth identification, as well as directional analysis of the illumination distribution.

In the remainder of the paper, Section 2 describes the framework for estimating illumination from shadows, and Section 3 introduces the skeleton cube as a reference object for the self shadow method. We compare the cast shadow method and the new self shadow method in Section 4, and show experimental results in Section 5. Section 6 concludes the paper.

\section{Illumination Estimation from Shadows}

In this section, we describe the framework for estimating illumination from shadows in a way that accommodates both the cast shadow method and the self shadow method. The lighting environment is approximated by a distribution of point light sources so that the problem is attributed to solve for the radiant intensity of each of discretely sampled sources. Although the distribution is first assumed to be at an infinite distance, we later take the distance into account.

By convention, we utilize the following terms - shadow image: the image with shadows, reference object: the object of known size which casts shadows, and shadow surface: the surface onto which the reference object casts shadows.

Note that the shadow surface is typically the same plane on which the reference object is placed in the cast shadow method, but it is the inner surface of the reference object such as the skeleton cube in the case of the self shadow method. Typical reference object in the cast shadow method is rectangular solid. Observing the radiance at sample points that are on the shadow surface, we estimate the lighting environment based on the following premises.

Camera: The camera that captures a shadow image including the reference object is calibrated so that we know the relationship of corresponding points in the scene and in the image. (The skeleton cube can be conveniently used for calibration as well).

Coordinate System: We define the origin of the world coordinate system at the bottom of the reference object in such a way that each axis of it is aligned parallel to the side of the skeleton cube. We locate the lighting environment and cameras that capture images in the world coordinate system.

\subsection{Reflectance Model}

We assume that the surface reflection of the reference object is described by the simplified Torrance-Sparrow model $[4,14]$ which can represent both diffuse and specular reflection, and it especially describes the specular reflection with physical properties. It is in this sense more general than other models such as the Phong reflectance model [8]. In this paper, we presume the influence of inter-reflection is ignorable and concentrate our discussion on the first reflection.

With the simplified Torrance-Sparrow model, observed radiance, $I(\boldsymbol{x})$, at the minute area around point $\boldsymbol{x}$ is described as

$$
I(x)=\left(k_{d} R_{d}+k_{s} R_{s}\right) L_{\mathcal{L}},
$$

where $L$ is the radiant intensity of light source $\mathcal{L}$. $R_{d}$ and $R_{s}$ denote the diffuse and the specular component of a bidirectional reflectance distribution function, respectively, whereas $k_{d}$ and $k_{s}$ are the weighting coefficients of them. Though $I(\boldsymbol{x})$ and $L$ are functions with respect to the wavelength, we utilize the functions at three wavelengths for red, green and blue. For the sake of a simple description, we do not denote it explicitly in the following.

Assuming the diffuse component is represented as Lambertian, we have $R_{d}=\boldsymbol{N} \cdot \boldsymbol{L} / r^{2}$, where $\boldsymbol{N}, \boldsymbol{L}$, and $r$ denote the surface orientation, the direction of light source $L$, and the distance between light source $L$ and point $\boldsymbol{x}$, respectively. The specular component, $R_{s}$, is represented as

$$
R_{s}=\frac{1}{r^{2}} \frac{1}{\boldsymbol{N} \cdot \boldsymbol{V}} \exp \left[-\frac{\left(\cos ^{-1}(\boldsymbol{N} \cdot \boldsymbol{H})\right)^{2}}{2 \sigma^{2}}\right],
$$

where $\boldsymbol{V}, \boldsymbol{H}$, and $\sigma$ denote the viewing direction, the half vector of $\boldsymbol{L}$ and $\boldsymbol{V}$, and the surface roughness, respectively.

The radiance of point $\boldsymbol{x}$ on the shadow surface can be generally formulated as

$$
I(x)=\sum_{i=1}^{N} \mathcal{M}\left(x, \mathcal{L}_{i}\right)\left(k_{d} R_{d}+k_{s} R_{s}\right) L_{\mathcal{L}_{i}},
$$

where $N$ is the number of point light sources, and $\mathcal{M}\left(\boldsymbol{x}, \mathcal{L}_{i}\right)$ is a mask term that encodes the self shadow of the skeleton cube. The mask term, $\mathcal{M}\left(\boldsymbol{x}, \mathcal{L}_{i}\right)$, is binary, indicating whether point 
$\boldsymbol{x}$ is illuminated by light source $\mathcal{L}_{i}$ or not, i.e., $\mathcal{M}\left(\boldsymbol{x}, \mathcal{L}_{i}\right)=1$ if light source $\mathcal{L}_{i}$ illuminates point $\boldsymbol{x}$ and $\mathcal{M}\left(\boldsymbol{x}, \mathcal{L}_{i}\right)=0$ otherwise.

\subsection{Computational Algorithm}

When we sample $M$ points on the shadow surface for observing the radiance, we have a matrix representation based on Equation (3)

$$
\left[\begin{array}{c}
I\left(\boldsymbol{x}_{1}\right) \\
I\left(\boldsymbol{x}_{2}\right) \\
\vdots \\
I\left(\boldsymbol{x}_{M}\right)
\end{array}\right]=\left[\begin{array}{cccc}
K_{11} & K_{12} & \cdots & K_{1 N} \\
K_{21} & K_{22} & \cdots & K_{2 N} \\
\vdots & \vdots & \ddots & \vdots \\
K_{M 1} & K_{M 2} & \cdots & K_{M N}
\end{array}\right]\left[\begin{array}{c}
L_{\mathcal{L}_{1}} \\
L_{\mathcal{L}_{2}} \\
\vdots \\
L_{\mathcal{L}_{N}}
\end{array}\right],
$$

where

$$
K_{m n}=\mathcal{M}\left(\boldsymbol{x}_{m}, \mathcal{L}_{n}\right)\left(k_{d} R_{d}+k_{s} R_{s}\right) .
$$

We then write Equation (4) simply as

$$
\boldsymbol{I}=\boldsymbol{K} \boldsymbol{L},
$$

where $\boldsymbol{K}=\left(K_{m n}\right)$. Given a sufficient number of surface radiance samples (i.e. $M \gg N$ ), it is in principle possible to solve Equation (6) for $\boldsymbol{L}$ by the linear least squares method with nonnegative variables,

$$
\min _{\boldsymbol{L}} \frac{1}{2}\|\boldsymbol{K} \boldsymbol{L}-\boldsymbol{I}\|_{2}^{2} \text { subject to } \boldsymbol{L} \geq 0 .
$$

Namely, we can obtain the radiant intensities in vector $\left[L_{\mathcal{L}_{1}}, L_{\mathcal{L}_{2}}, \ldots, L_{\mathcal{L}_{N}}\right]^{\top}$.

\section{Reference Object in the Self Shadow Method}

In order to estimate the parameters of light sources in the self shadow method, we analyze self shadows of a reference object. Given that we can utilize a reference object of known shape, we design the skeleton cube, a hollow cube which casts shadows to its inside surface, as shown in Figure 2. The design is on the basis of the two requirements that are inconsistent to each other. Namely, the shape should be simple while some complexity is desirable:

- Simplicity: Simple shape is suitable for the computational costs. Then, a large portion of the surface should be observable in sampling the surface intensity.

- Complexity: The shape needs to be complex to a certain extent so that self shadows occur under variable lighting conditions.

We employ the skeleton cube as a reference object that satisfies the above requirements. That is, under light sources at almost any positions in a scene, it casts shadows on its inside surfaces and the shadows can be observed from any viewpoints. See also Appendix on how the self shadows are generated under light sources at various locations.

The skeleton cube can also be used as a reference object for geometric calibration of cameras, which is an ordinary method by matching corresponding points in a captured image and the model although we do not go into the details of the geometric calibration in this paper.

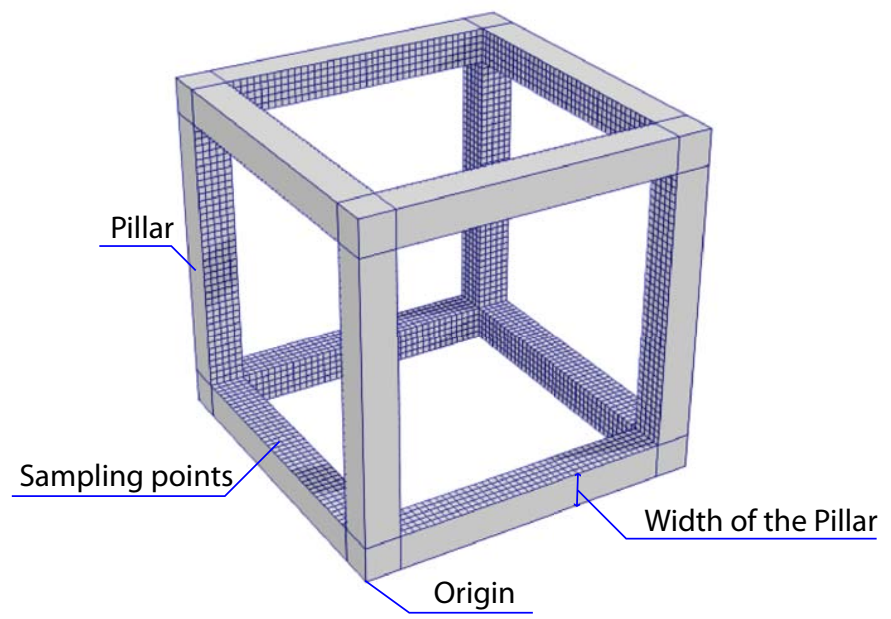

Figure 2: The skeleton cube. Sampling points are at the lattice positions on the inside surface of the cube.

\section{Comparisons of the Self Shadow Method and the Cast Shadow Method}

Our basic strategy for estimating illumination distribution in the $3 \mathrm{D}$ space is twofold, i.e., directional analysis and distance identification. We first assume an illumination distribution on a hemisphere that has a certain radius, and estimate the radiant intensities of the light sources by solving Equation (6). Then, by repeating the estimate of the radiant intensities while decreasing the radius step-by-step, we can identify the directions along which the estimated radiant intensities remain high at all the distances as those of major light sources in the scene. Through this process, light sources with weak radiant intensities such as reflections from the walls are regarded as ambient lighting.

In order to explicitly identify the light sources in the 3D space, we also need to know the distances to them, for example from the center of the reference object, the origin of the world coordinate system. This is important especially when there are near light sources in the scene. For this purpose, we can investigate possible locations of the light sources on lines along the above estimated directions. In practice, we consider distributions of light sources at a certain interval along the lines and then solve Equation (6) for $\boldsymbol{L}$, analogously as in the directional analysis. Note that it is useful to retain the assumption of an illumination distribution on the hemisphere for describing the ambient light.

Finally, we refine the estimates of light source distribution by again solving Equation (6), but for $\boldsymbol{L}$ that represents light sources which we assume in the vicinity of the once identified locations of light sources.

We now discuss the stability issues for each of the directional analysis and the distance identification while comparing the self shadow method with the cast shadow method. 
Light source in stable direction

Light source in unstable direction

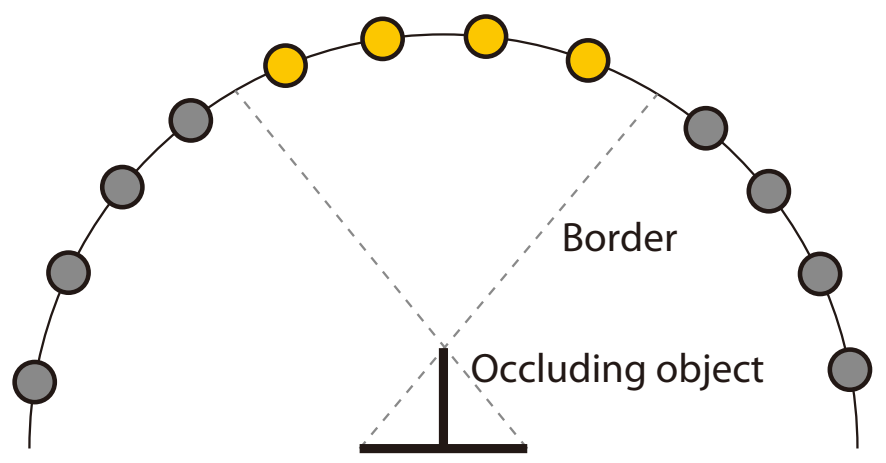

Sampling area

Figure 3: One-dimensional sketch that shows directions of light sources which can be stably estimated by the cast shadow method.

\subsection{Directional Analysis}

The cast shadow method: In [10], several issues in the cast shadow method are pointed out and some of them have been alleviated. Possible instability of estimation is due to factors such as what size portions of shadows cast by an object can be observed in the input image, and what size portions of the view of the camera are occluded by the object.

However, an important related factor which has not yet been discussed is that there may be light sources whose information cannot be reflected in the sampling area of the shadow surface. A simple one-dimensional sketch in Figure 3 suffices to illustrate it. In the figure, although the four discretely sampled light sources on the top of the hemisphere cast shadows partly to the sampling area behind the occluding reference object, it is not the case for the remaining eight sources at both sides. For example, all the four sources that illuminate the scene from the left side would cast shadow on the entire sampling area behind the object, and illuminate the front side in an equivalent way. It is therefore obvious that the analysis of the shadows would not give precise estimates of the radiant intensities of those light sources.

The self shadow method: The self shadow method on the other hand allows those light sources at both sides to be identified thanks to multiple shadow surfaces with different orientations which span the three dimensional space. As has been clarified in [10], what matters to realize a stable solution is not the size of sampling area, but variations of observable image brightnesses inside shadow surfaces which are derived from combinations of different light sources. This supports the advantage of the self shadow method although the area of the inner shadow surfaces of the skeleton cube tends to be smaller than the case of the cast shadow method.

Comparisons: To see the relevancy of the above discussion, we study how accurately the radiant intensities can be actually
Table 1: Average errors and standard deviations in estimating the radiant intensities (See also Figure 4)

\begin{tabular}{lrr}
\hline angle & cast shadow & self shadow \\
\hline $10-35$ & $6.78 \mathrm{e}-07,9.67 \mathrm{e}-07$ & $6.78 \mathrm{e}-07,9.67 \mathrm{e}-07$ \\
$20-55$ & $2.88,5.59$ & $9.5 \mathrm{e}-07,1.14 \mathrm{e}-06$ \\
$45-80$ & $3.56,6.88$ & $7.25 \mathrm{e}-07,1.03 \mathrm{e}-06$ \\
$10-80$ & $2.42,3.90$ & $1.16 \mathrm{e}-06,1.13 \mathrm{e}-06$ \\
\hline border $=36.9^{\circ}$ & &
\end{tabular}

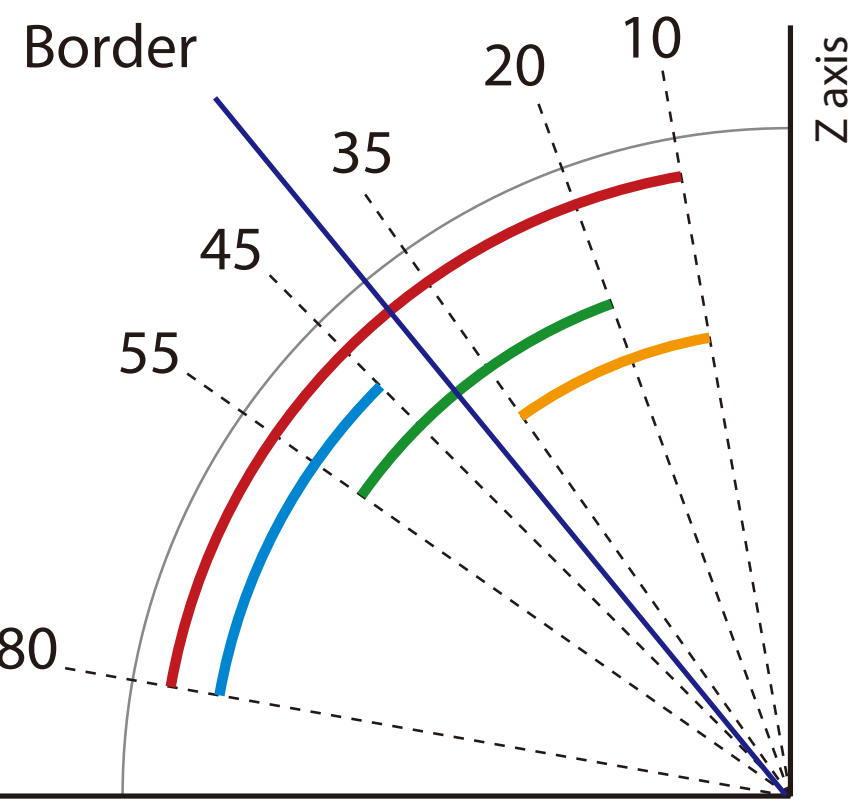

XY plane

Figure 4: Schematic for evaluating the directional analysis. Four arcs are corresponding to the four rows in Table 1.

recovered by the two methods in terms of average errors and standard deviations through simulations using such illumination distributions as sketched in Figure 3. The radiant intensities to be estimated are randomly set in a way that the average value is normalized to 1.0 .

Table 1 shows the results in the cases of estimating four different partial distributions of the light sources which are located at every five degrees as depicted in Figure 4. For example, the first row of the table (angle 10-35) corresponds to the case that all the light sources are above the angle at the border (angle 36.9). The cast shadow method as well as the self shadow method should provide stable estimates in this case, and the estimated intensities are in fact very close to the true value with only very small errors. In the other three cases, however, both average errors and the standard deviations by the cast shadow method are very large, which is natural according to the above analysis, whereas those by the self shadow method are kept small, reflecting its higher stability. 


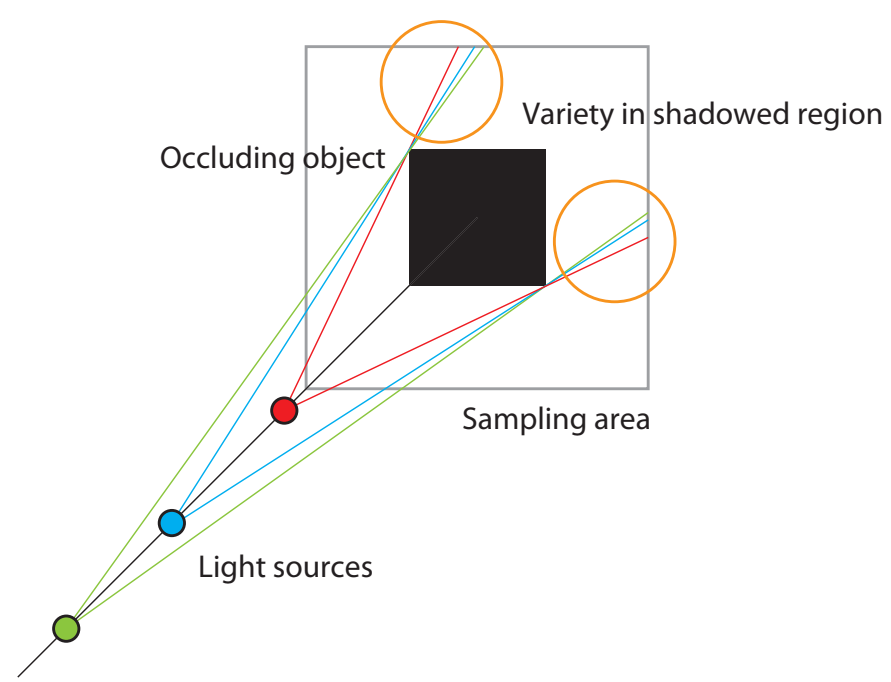

Figure 5: A top view of the cast shadow method. Shadows cast by light sources in an identical direction but at different distances are sketched. The circles are placed to highlight possible differences between the shadows.

\subsection{Distance Identification}

The cast shadow method: Figure 5 shows a top view of the cast shadow method where shadows cast by different light sources are sketched on the top of each other. The light sources are located in an identical direction but at different distances. To be noted is that the variation between them is relatively small (see the marked areas with circles) as the shadows are cast on a single planar surface. Thus, the chance of successfully sampling varieties of brightnesses is quite low, which is intrinsic to the method and probably the reason why the notion of the distance has not so far been well discussed in the framework.

The self shadow method: Figure 6 shows a configuration of the self shadow method. It is synthesized by CG using two light sources that are located in an identical direction but at different distances. In the marked parts with the circles we can observe shadows cast to the inner surfaces. Among those, the shadows by the two light sources due to the adjacent occluding pillar (marked with the smaller circle) are almost identical as was the case with the cast shadow method. On the other hand, the shadows due to the diagonal pillar (marked with the bottomright circle) is of importance in the presence of near light sources because the variations are more significant, which help estimating the distances.

Comparisons: As has been already discussed, the self shadow method allows a higher chance for successfully sampling varieties of brightnesses. It is again due to the fact that it has multiple shadow surfaces with different orientations, and also that some shadows are cast from distant pillars, which give rise to more variations of brightnesses in shadows.

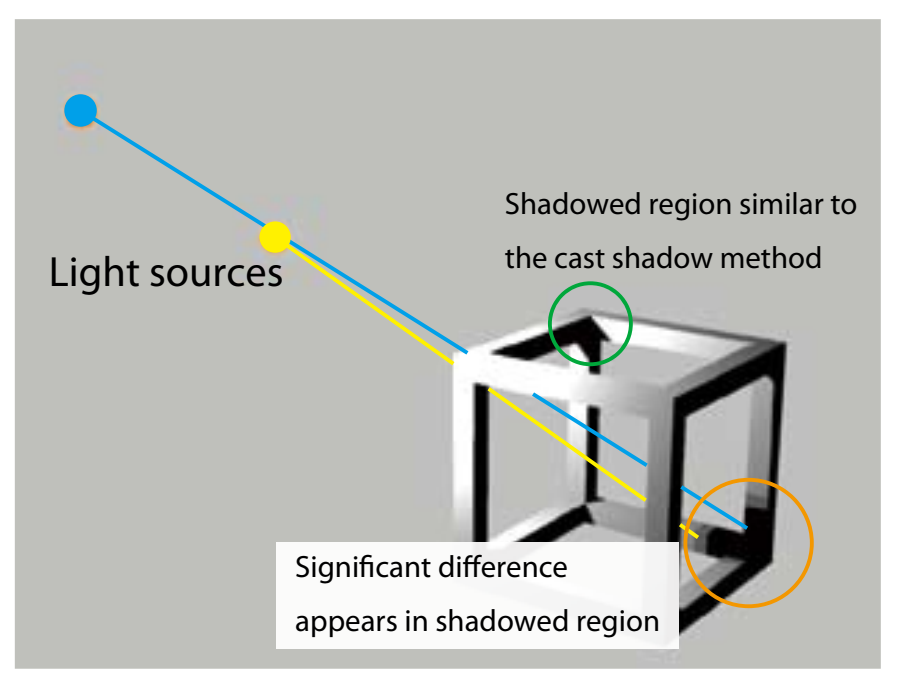

Figure 6: A view of the self shadow method. Shadows cast by the two light sources in an identical direction but at different distances are generated by $C G$. The circles are placed to highlight differences between the self shadows.

\section{Experiments}

\subsection{Performance Evaluation}

We evaluate the performances of the self shadow method and the cast shadow method for estimating near lighting in the following configuration. See also Figure 7.

- Camera: Dragonfly2 (Point Gray Research Inc.), 1024 $\times 768$ pixels, color CCD (Captured images are shown in Figure 8).

- Lighting Environment: Four candles are closely located to the reference objects (two of them on the right appear merged as they are placed in tandem along the viewing direction), and four florescent light tubes are on the ceiling.

- Reference objects:

- The cast shadow method: We place a black rectangular solid (size: $50 \times 100 \times 200 \mathrm{~mm}$ ) on a white Lambertian planar surface from which we sample the radiant intensities.

- The self shadow method: We employ the skeleton cube (size: $100 \times 100 \times 100 \mathrm{~mm}$ with $10 \mathrm{~mm}$ pillar) whose surface is nearly Lambertian and mutual reflections between the pillars are ignorable.

The first experiment is for comparison; we estimate the lighting environment by the cast shadow method that was proposed for estimating directional light sources in [12]. We then observe if any artifact is caused by applying the method to the near lighting. Next, we apply the cast shadow method and the self shadow method, respectively to the scene with near light sources, and show how the new self shadow method successfully estimates complex near lighting. 

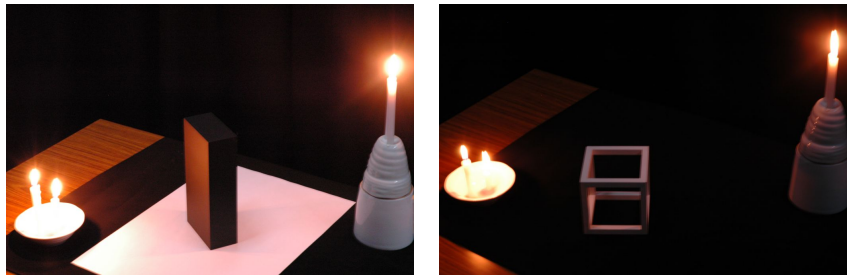

(a) Cast shadow method

(b) Self shadow method

Figure 7: Configuration of experiments

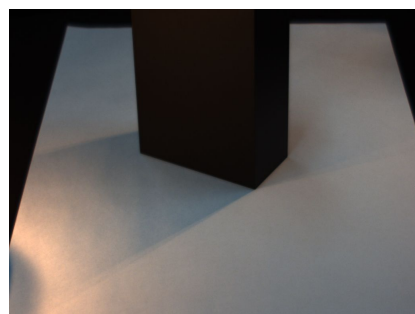

(a) Cast shadow method

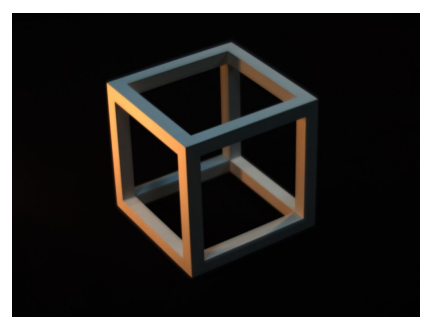

(b) Self shadow method
Figure 8: Captured images

The cast shadow method with distant lighting model: We model the distant lighting by a distribution of directional light sources. They are directed towards the center from the vertices of an upper hemisphere of the dodecahedron whose faces are recursively subdivided (1280-hedron, 301 vertices). Figure 9 shows the results of the estimations where the pseudo-colors of the dome indicate the radiant intensities.

Some false radiant intensities appear close to the frontal fringe of the hemisphere and they are caused by the lack of varieties of brightness in the shadows as discussed in Section 4.2. Figure 10 illustrates the captured intensities and the relit intensities which are synthesized by using the estimated lighting. The figure shows that the left-bottom bright region is not properly relit by the estimated distant lighting, and that a false cast shadow appears in the left-top region behind the rectangular block, which indicates that light sources that never exist in the scene have been mistakenly estimated. The average errors of pixel values are $(0.21,0.12,0.07)$ in $(R, G, B)$ values.

The cast shadow method with near lighting model: To estimate near lighting, we first need to find directions of primary light sources by the directional analysis. We utilize the same polyhedron that is described for the previous experiment by considering the radius ranging from $1000 \mathrm{~mm}$ down to 250 $\mathrm{mm}$ at a interval of $10 \mathrm{~mm}$. Figure 11 illustrates the results of the directional analysis. Figure $11 \mathrm{~b}$ shows that the left-bottom light source is detected but the right-top light source is not. Again, this can be regarded as caused by the issues which we explained in the previous sections, and shows the difficulty in estimating near light sources.

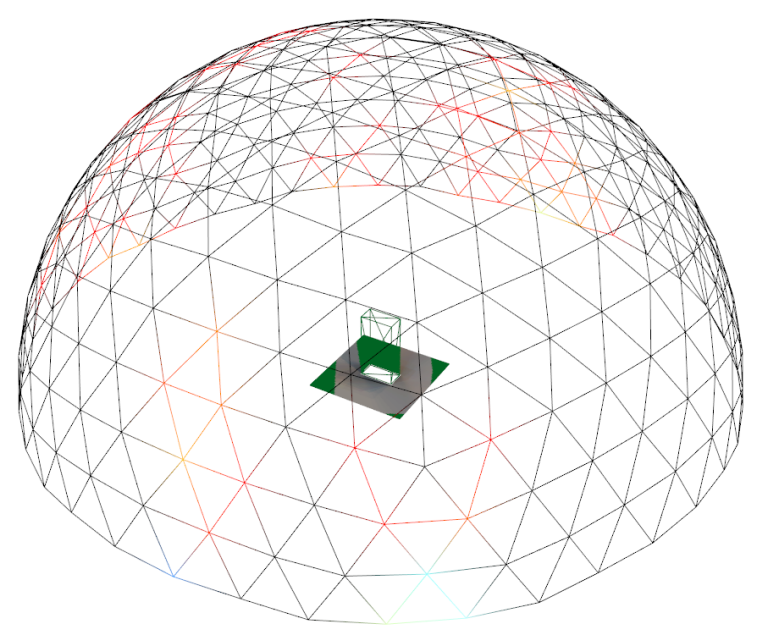

Figure 9: Estimated distant lighting by the cast shadow method. The vertices of the dome denote the directional light sources, and the pseudo-colors of the dome indicates the radiant intensities of the light sources with the color of black-body radiation. The occluding object and the sampling plane are placed in the center-bottom of the dome.

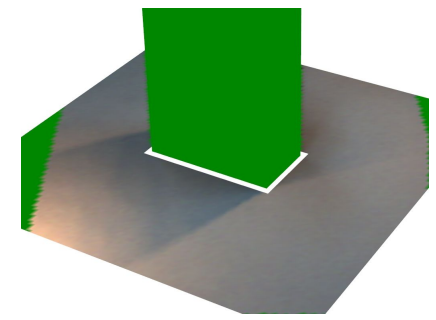

(a) Captured intensities

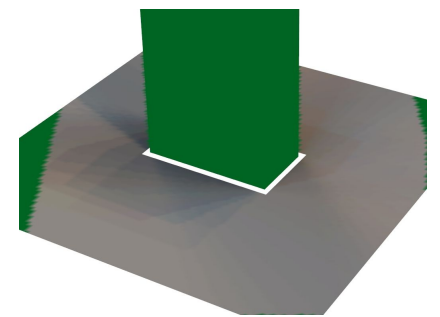

(b) Relighted intensities using the estimated lighting
Figure 10: Qualitative evaluation of the cast shadow method with distant lighting. Green pixels denote the occluding object and regions outside sampling area.

The self shadow method with near lighting model (the proposed): Figure 12 illustrates the results of the directional analysis which we carried out analogously as in the previous two experiments. Unlike the cases with the cast shadow method, the right-top light source is successfully estimated as well as the one in the left bottom. Figure 13 shows the resulting light source distribution that is estimated by the directional and then the depth identification. The points with small circles close to the skeleton cube are the detected near light sources by the depth identification along to the detected directions. They reflect the candles that are found in Figure 7b. Finally, Figure 14 compared the captured intensities and the relit intensities using the estimated lighting. The figure shows that the relit intensities of the skeleton cube are very close to those originally captured. The average errors of the pixel values are $(0.16,0.10$, $0.08)$ in $(R, G, B)$ values. 


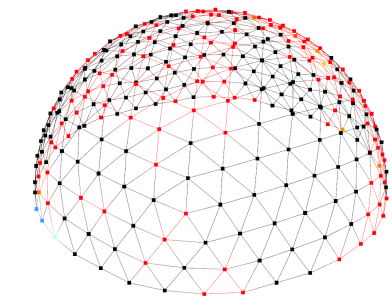

(a) Estimated radiant intensity

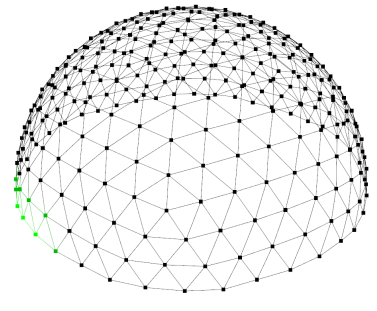

(b) Detected direction of estimated primary light sources

Figure 11: Detected directions of light sources by the directional analysis by the cast shadow method. The pseudocolors of the dome in (a) indicate the radiant intensities of the light sources with the color of black-body radiation. The green vertices in (b) denote the directions of the detected light sources.

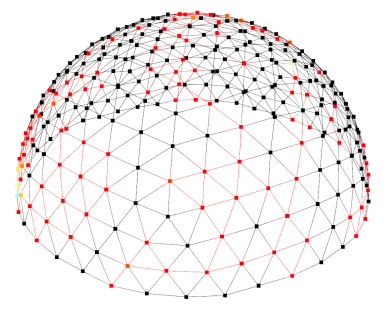

(a) Estimated radiant intensity

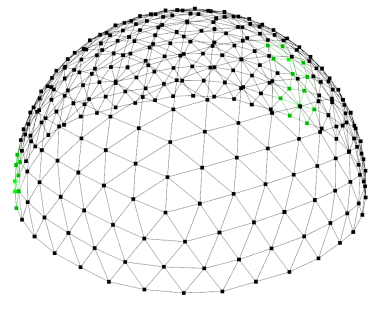

(b) Detected direction of estimated primary light sources
Figure 12: Detected directions of light sources by the directional analysis with the self shadow method. The pseudo-colors of the dome in (a) indicate the radiant intensities of the light sources with the color of black-body radiation. The green vertices in (b) denote the directions of the detected light sources.

\subsection{CG Generation of Temporal Sequence}

By using a temporal image sequence of a scene we demonstrate the effectiveness of our method for estimating dynamic near lighting that is described in Section 5.1. Figure 15 shows the captured image sequence which illustrates the time varying effect of the candles. Figure 16 shows a virtually generated image sequence of a virtual skeleton cube that is illuminated by the estimated lighting environment. We can observe that detailed shadows and the highlighted regions on the pillars are appropriately synthesized since the complex illumination is successfully estimated. Finally, in Figure 17, we show an example of virtual illumination with an image sequence which is rendered with a CG model under the estimated lighting environment. While the expression of flames is usually difficult and requires relatively higher skills of modeling, we can easily obtain a distribution of positions and intensities of real flames from an image sequence.

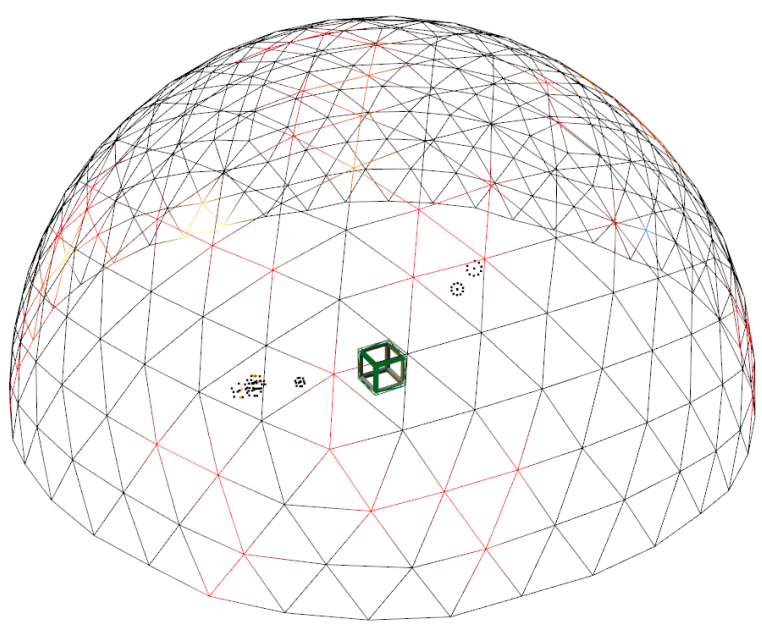

Figure 13: Estimated distant lighting by the self shadow method. The vertices of the dome denote the directional light sources, and points inside the dome denote the near light sources that are estimated by the distance identification. The pseudo-colors of the dome and the points indicate the radiant intensities of the light sources with the color of black-body radiation. The skeleton cube is shown in the center-bottom of the dome.

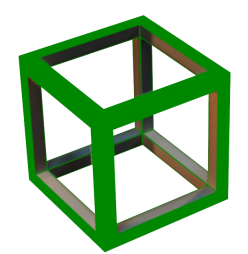

(a) Captured intensities

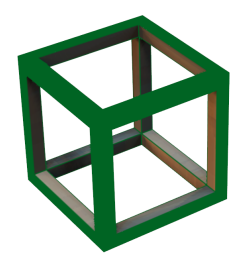

(b) Relighted intensities using the estimated lighting
Figure 14: Qualitative evaluation of the self shadow method with near lighting. Green pixels denote outer surfaces that are not for sampling.

\section{Conclusions}

We have proposed the self shadow method for estimating illumination distributions of a real scene. It utilizes variations of image brightnesses inside shadow surfaces of an occluding object itself. Through analytical comparisons to the conventional cast shadow method, we have shown why the self shadow method allows more stable estimation of illumination distributions, in particular in the presence of near light sources. We have also demonstrated through experiments that it works effectively with our twofold algorithm which consists of directional analysis and distance identification for estimating dynamic light sources including their proximity, which has been difficult in the previous related approach. For example, direct methods such as using a light probe or a fish-eye lens rely on high dynamic range images. Since they require multiple images of a static scene captured with 


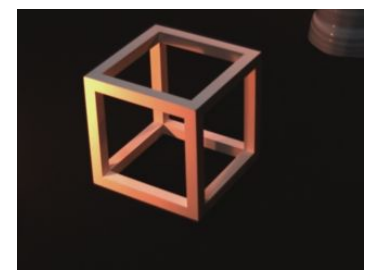

(a) \#0

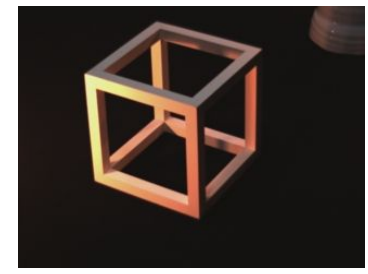

(f) \# 25

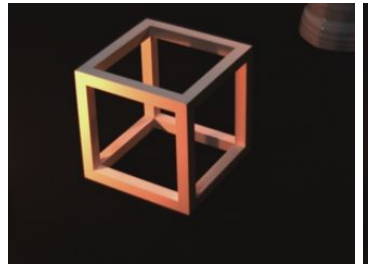

(b) \# 5

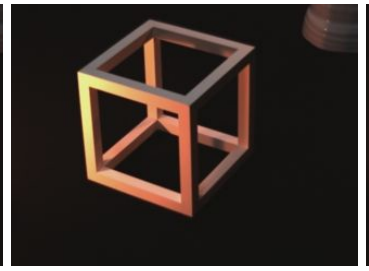

(g) \# 30

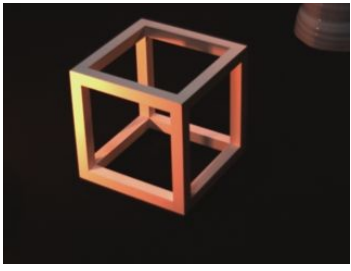

(c) \# 10

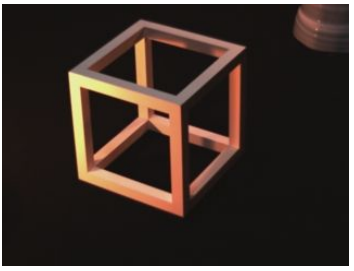

(h) \# 35

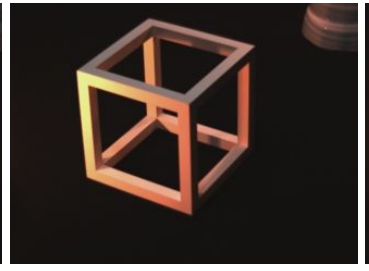

(d) \# 15

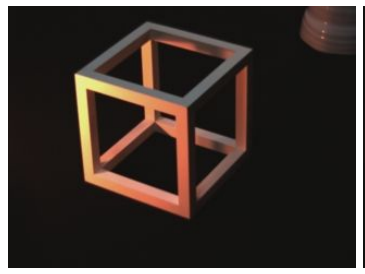

(i) \# 40

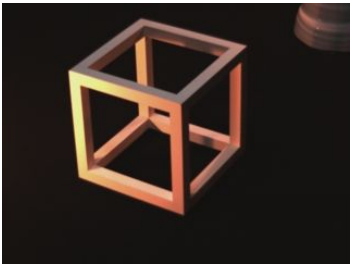

(e) \# 20

Figure 15: Captured image sequence. The images are captured at 15 frames per second.

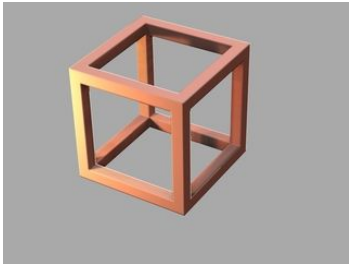

(a) \# 0

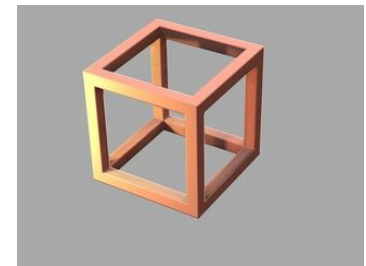

(f) \# 25

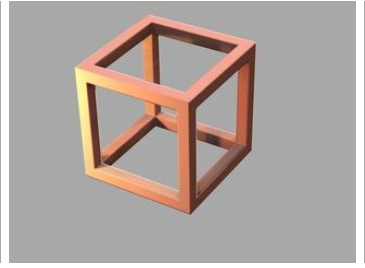

(b) \# 5

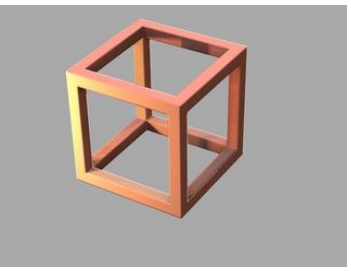

(c) \# 10

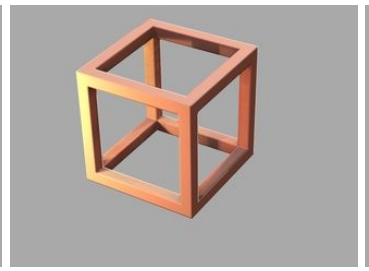

(d) \# 15

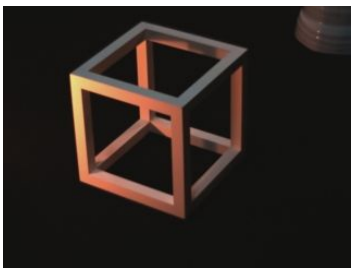

(j) \# 45

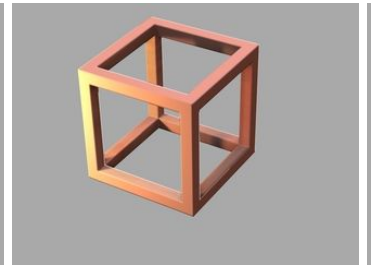

(g) \# 30

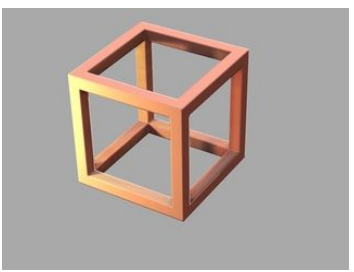

(h) \# 35

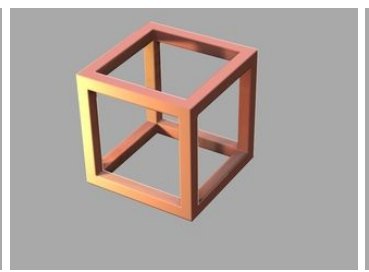

(i) \# 40

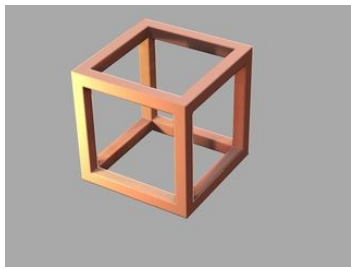

(e) \# 20

Figure 16: Generated image sequence of the skeleton cube with the estimated illumination distribution.

different levels of exposure or aperture, estimating the distance of dynamic lighting is not an easy task for a method using a stereo pair of fish-eye lenses.

Although the skeleton cube has been employed for the initial introduction of the self shadow method in this paper, other appropriate designs for reference objects may exist. The design should highly affects the accuracy of estimated light sources distribution. One of the keys to obtain an appropriate design is to analyse $\mathcal{M}$ in matrix $K$ which influences the stability of the estimation. Finally, a viewpoint from which we capture the object is also an important factor so that we observe a large variation of self shadows.

\section{Acknowledgement}

The work is supported by "Foundation of Technology Supporting the Creation of Digital Media Contents" project (CREST, JST) and Ministry of Education, Culture,
Sports, Science and Technology under the Leading Project, "Development of High Fidelity Digitization Software for Large-Scale and Intangible Cultural Assets".

\section{A Evaluating Capability of Self Shadow Generation of the Skeleton Cube}

In order to examine the occurrence of self shadows, we count the number of sampling points on the inside surface of the skeleton cube that are self occluded by other pillar of the hollow cube when viewed from each possible point light source. We consider the space for the point light sources to be placed, ranging from $(-1500,-1500,0)$ to $(1500,1500,3000)$ with (spacing 100).

When a certain sampling point is self occluded viewing from a certain grid point, self shadow is cast on the point by a light source on the grid point. Hence, it would be a problem if there were only few points that are self occluded, since in that 


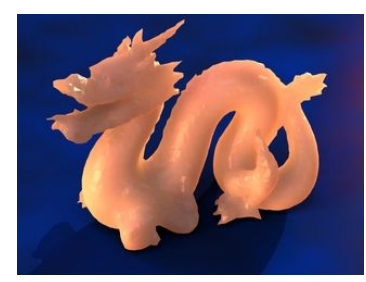

(a) \# 0

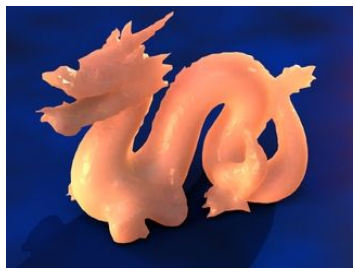

(f) \# 25

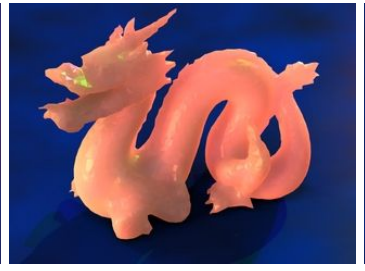

(b) \# 5

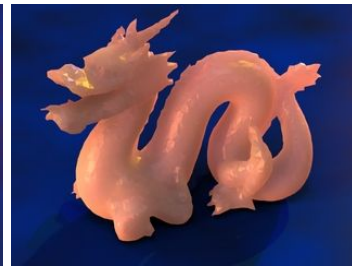

(g) \# 30

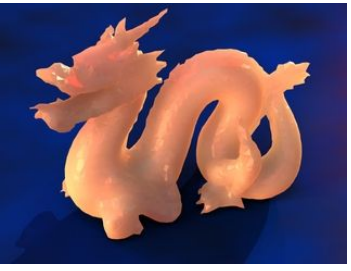

(c) \# 10

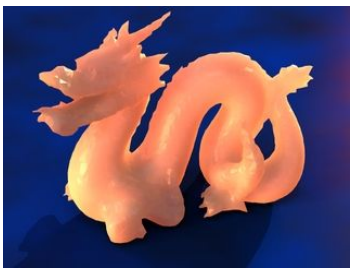

(h) \# 35

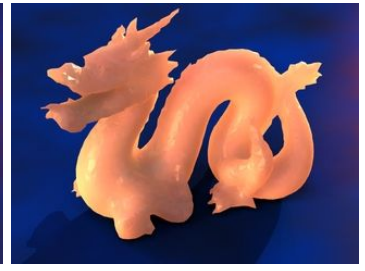

(d) \# 15

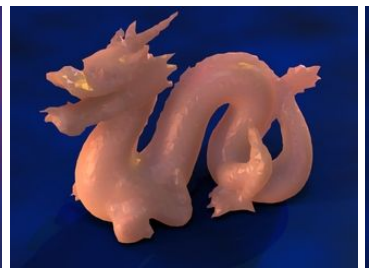

(i) \# 40

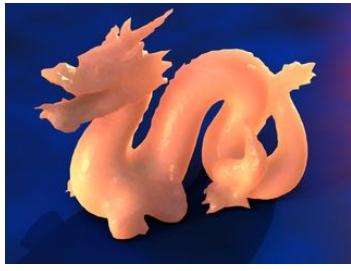

(e) \# 20

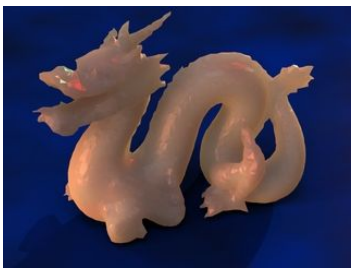

(j) \#45

Figure 17: Generated image sequence of a dragon with the estimated illumination distribution. The model of a dragon is distributed by the Stanford Computer Graphics Laboratory.

case almost all the $\mathcal{M}$ term in Equation (5) would equal to 1 , and thus the estimation of radiant intensities might become unstable. Therefore, it is a desirable condition for the skeleton cube that it has self shadows to some extent wherever the point light sources are placed arbitrarily.

We show some results of verification in Figure 18. The color of each position on the planes in Figure 18 signifies the possible number of self occlusion by a point light source that is placed on that position of the place. The color denotes the number, i.e. red denotes a low, and blue denotes a high number. For the total number of sampling points, 5300, even the minimum number of occluded points was 704 (when viewed from $V_{\text {min }}$ in Figure 18) whereas the maximum was 3540. This indicates that the skeleton cube has self shadows of some good extent by point light sources at all the considered positions, which is a suitable characteristic for a reference object as a probe of lighting environment.

\section{References}

[1] P. Debevec. Rendering synthetic objects into real scenes: Bridging traditional and image-based graphics with global illumination and high dynamic range photography. In ACM SIGGRAPH, pages 189-198, 1998.

[2] D. Frolova, D. Simakov, and R. Basri. Accuracy of spherical harmonic approximations for images of Lambertian objects under far and near lighting. In European Conference on Computer Vision, pages 574587, 2004.

[3] K. Hara, K. Nishino, and K. Ikeuchi. Determining reflectance and light position from a single image without distant illumination assumption. In IEEE International Conference on Computer Vision, pages 560-567, 2003.
[4] K. Ikeuchi and K. Sato. Determining reflectance properties of an object using range and brightness images. IEEE Transactions on Pattern Analysis and Machine Intelligence, 13(11):1139-1153, 1991.

[5] S. R. Marschner and D. P. Greenberg. Inverse lighting for photography. In Fifth Color Imaging Conference, pages 262-265, 1997.

[6] P. Nillius and J.-O. Eklundh. Automatic estimation of the projected light source direction. In IEEE Conference on Computer Vision and Pattern Recognition, volume I, pages 1076-1083, 2001.

[7] A. P. Pentland. Finding the illumination direction. Journal of Optical Society of America, 72(4):448-455, 1982.

[8] B. Phong. Illumination for computer generated pictures. In Communications of the ACM, pages 311-317, 1975.

[9] M. W. Powell, S. Sarkar, and D. Goldgof. A simple strategy for calibrating the geometry of light sources. IEEE Transactions on Pattern Analysis and Machine Intelligence, pages 1022-1027, 2001.

[10] I. Sato, Y. Sato, , and K. Ikeuchi. Stability issues in recovering illumination distribution from brightness in shadows. In IEEE Conference on Computer Vision and Pattern Recognition, pages 400-407, 2001.

[11] I. Sato, Y. Sato, and K. Ikeuchi. Acquiring a radiance distribution to superimpose virtual objects onto a real scene. IEEE Transactions on Visualization and Computer Graphics, 5(1):1-12, 1999.

[12] I. Sato, Y. Sato, and K. Ikeuchi. Illumination from shadows. IEEE Transactions on Pattern Analysis and Machine Intelligence, 25(3):290-300, 2003. 


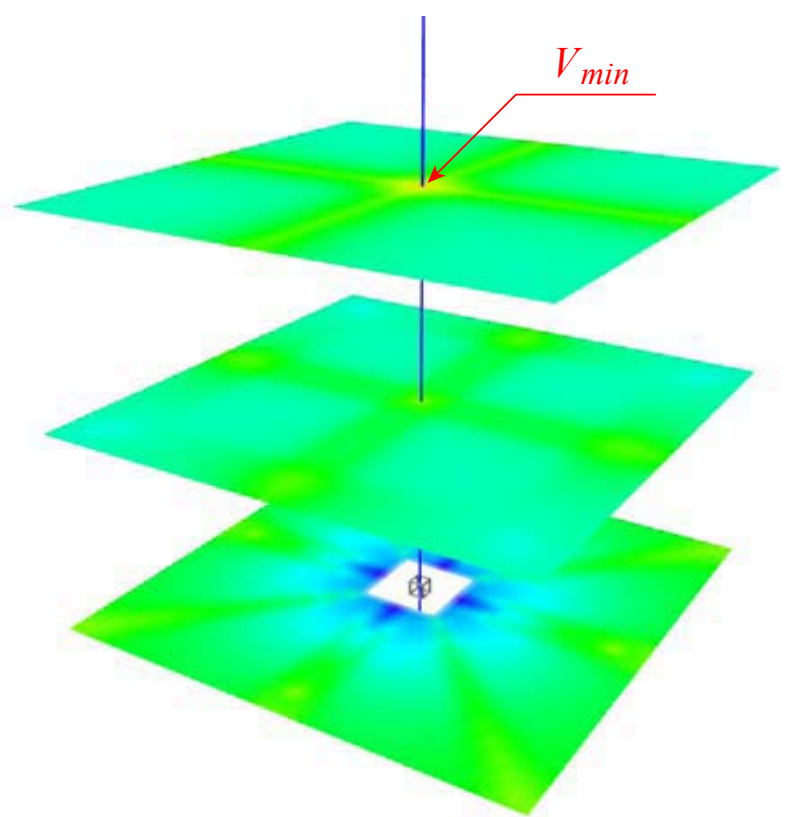

Figure 18: Self occlusion of the skeleton cube. We count the number of sampling points on the inside surface of the skeleton cube that are self occluded by other pillar of the hollow cube when viewed from each possible $3 D$ grid position for a point light source. The color corresponds to the number of self occluded points: red for low, blue for high numbers.

[13] T. Takai, K. Niinuma, A. Maki, and T. Matsuyama. Difference sphere: An approach to near light source estimation. In IEEE Conference on Computer Vision and Pattern Recognition, volume I, pages 98-105, 2004.

[14] K. E. Torrance and E. M. Sparrow. Theory for offspecular reflection from roughness surface. Journal of the Optical Society of America, 57:1105-1114, 1967.

[15] Y. Wang and D. Samaras. Estimation of multiple illuminants from a single image of arbitrary known geometry. In European Conference on Computer Vision, pages 272-288, 2002.

[16] Y. Yang and A. Yuille. Sources from shading. In IEEE Conference on Computer Vision and Pattern Recognition, pages 534-539, 1991.

[17] Y. Zhang and Y. Yang. Multiple illuminant direction detection with application to image system. IEEE Transactions on Pattern Analysis and Machine Intelligence, 23:915-920, 2001.

[18] Q. Zheng and E. Chellappa. Estimation of illuminant direction, albedo, and shape from shading. IEEE Transactions on Pattern Analysis and Machine Intelligence, 13(7):680-702, 1991.
[19] W. Zhou and C. Kambhamettu. Estimation of illuminant direction and intensity of multiple light sources. In European Conference on Computer Vision, pages 206$220,2002$. 